
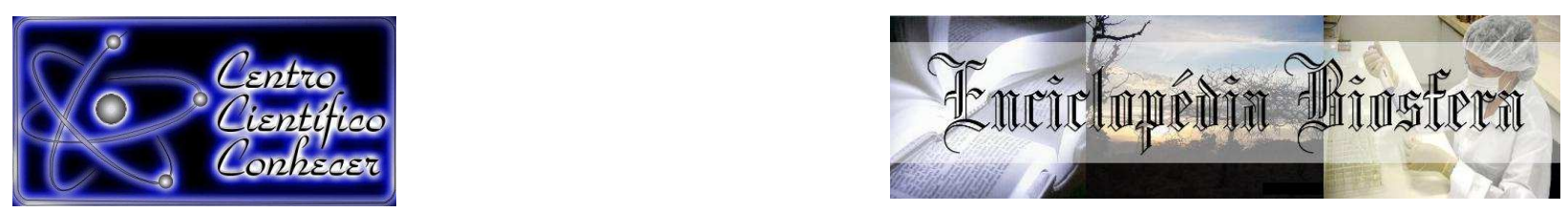

\title{
AVALIAÇÃO DO CRESCIMENTO DE CLONES DE EUCALIPTO NO NORTE DE
} MINAS GERAIS

\author{
Juscelina Arcanjo dos Santos ; Vinícius Orlandi Barbosa Lima²; Jéssica Costa de \\ Oliveira $^{3}$; Priscila Silva Matos ${ }^{4}$; Paulo André Trazzi ${ }^{2}$ \\ ${ }^{1}$ Mestre em Ciências Florestais, Universidade Estadual Sudoeste da Bahia \\ (celinarcanjo@hotmail.com), Vitória da Conquista, Bahia \\ 2Professor, Instituto Federal de Educação do Norte de Minas Gerais, Departamento de \\ Engenharia Florestal \\ ${ }^{3}$ Engenheira Florestal, Instituto Federal de Educação do Norte de Minas Gerais, \\ Departamento de Engenharia Florestal \\ ${ }^{4}$ Mestre em Ciências Florestais, Universidade Estadual Sudoeste da Bahia
}

Recebido em: 02/10/2017 - Aprovado em: 21/11/2017 - Publicado em: 05/12/2017 DOI: 10.18677/EnciBio_2017B7

\section{RESUMO}

A busca por qualidade e produtividade nos povoamentos florestais tem demandado pesquisas à procura de materiais genéticos mais produtivos e adaptados às diferentes condições ambientais. A identificação de clones capazes de se estabelecer e desenvolver em condições de deficiência hídrica no solo é um desafio para as empresas florestais, pois estes fatores são de grande relevância para o êxito de um povoamento florestal. Sendo assim, o objetivo deste trabalho foi avaliar as características de crescimento de três materiais genéticos de Eucalyptus urophylla no Norte de Minas Gerais. O estudo foi conduzido em um plantio de Eucalyptus urophylla, com 5 anos de idade, localizado no município de Águas Vermelhas, Minas Gerais, Brasil. Foram avaliados 3 clones: AEC 144, GG100 e AEC 224, comumente destinados como matéria prima para a produção de carvão vegetal. Para a realização do inventário florestal foi medido a circunferência à altura do peito (CAP) e altura de todas as árvores da parcela. O método utilizado para a cubagem rigorosa foi o método de Smalian, sendo cubadas quatro árvores por classe de diâmetro por clone. Na comparação entre as médias dos clones realizada a partir do teste t foi observada diferença significativa para todas as variáveis estimadas. Os clones de Eucalyptus urophylla tiveram incremento médio anual (IMA) satisfatório quando comparados a resultados obtidos na mesma região de estudo, demonstrando boa adaptação às condições ambientais. Desta forma, o uso desses clones em plantios comerciais pode proporcionar boa produtividade.

PALAVRAS-CHAVE: Condições climáticas, Materiais genéticos, Produtividade

\section{GROWTH EVALUATION OF EUCALYPTUS CLONES IN NORTHERN MINAS GERAIS}

\section{ABSTRACT}

The demand for quality and productivity in forest stands has required research on productive genetic material and adapted in different environmental conditions. The identification of clones capable of establishing and developing water-deficient soil conditions is a challenge for forestry companies because these factors are of great relevance for the success of a forest. Thus, the objective of this work was to evaluate 
the growth characteristics of three Eucalyptus urophylla genetic materials in the Northern Minas Gerais. The study was conducted on a 5-year-old plantation of Eucalyptus urophylla, located in Águas Vermelhas, Minas Gerais, Brazil. Three clones were evaluated: AEC 144, GG100 and AEC 224, commonly used as source to charcoal production. The forest inventory was measured by the circumference at breast height (CAP) and the height of all the trees in the plot. The volume was estimated by stem rigorous scaling and cylindrical volume by Smalian method, with four trees per diameter class per clone. In the comparison between the means of the clones performed by $t$ test, a significant difference was observed for all variables estimated. Eucalyptus urophylla clones had a satisfactory mean annual increment (IMA) when compared to the results obtained at the same study region, showing good adaptation to environmental conditions. In this way, the use of these clones in commercial plantations may provide good productivity.

KEYWORDS: Climate conditions, Genetic materials, Productivity

\section{INTRODUÇÃO}

Eucalyptus é o gênero florestal mais plantado no Brasil, ocupando uma área superior a 5,7 milhões de hectares de árvores plantadas do país que estão localizadas, principalmente, em Minas Gerais (24\%), em São Paulo (17\%) e no Mato Grosso do Sul (15\%) (IBA, 2017). A madeira de eucalipto é destinada à diversos segmentos do setor florestal como indústrias de celulose e papel, produção de carvão vegetal, geração de energia, manufatura de painéis, postes, estacas, dormentes, escoras e laminados (VENTURIN et al., 2013).

Em função dessa grande demanda por madeira as áreas de plantios clonais de Eucalyptus vêm sendo ampliadas cada vez mais em todo o território brasileiro, principalmente em decorrência das vantagens do processo de clonagem como a possibilidade de uniformidade dos plantios florestais e maximização da produtividade (ROSADO et al., 2012).

A busca por maior qualidade e produtividade nos povoamentos florestais tem demandado pesquisas à procura de materiais genéticos mais produtivos e adaptados às diferentes condições ambientais. A identificação de clones capazes de se estabelecerem e se desenvolverem em condições de deficiência hídrica no solo é um desafio para as empresas florestais, pois estes fatores são de grande relevância para o êxito de um povoamento florestal (TATAGIBA et al., 2016).

Alguns trabalhos estão sendo desenvolvidos com intuito de demonstrar o crescimento de diferentes genótipos de eucalipto e assim indicar os clones mais adequados a diversas condições ambientais (XAVIER et al., 2013; TATAGIBA et al., 2015). Estes trabalhos têm sido base para as recomendações de clones em áreas com déficit hídrico, proporcionando melhor qualidade dos povoamentos, maior produtividade e menores custos.

Dentre os fatores ambientais que podem ocasionar estresse nas plantas podem ser citados a radiação solar, disponibilidade hídrica e temperatura, sendo a água o fator mais limitante para a produtividade, visto que a deficiência hídrica afeta significativamente os processos fisiológicos (TAIZ; ZEIGER, 2013). Por ser o principal causador de perdas na produtividade, a utilização de materiais genéticos mais tolerantes ao estresse hídrico aliado a boas práticas de manejo constituem alternativas para a implantação de povoamentos florestais em regiões com baixos índices pluviométricos.

A região do Norte de Minas Gerais é caracterizada por apresentar um clima severo com temperaturas elevadas e baixa precipitação anual distribuída em um 
curto período do ano (FERNANDES, 2002). Nesta região há uma grande extensão de florestas plantadas com espécies do gênero eucalipto, sendo necessários estudos direcionados a identificação de materiais genéticos que apresentem melhor adaptabilidade às condições edafoclimáticas locais e com maiores índices de produtividade.

Portanto, diante da importância de obter informações precisas sobre a silvicultura do eucalipto no Norte de Minas Gerais, o objetivo deste trabalho foi avaliar as características de crescimento e produtividade de três materiais genéticos de Eucalyptus urophylla S. T. Blake no Norte de Minas Gerais em um sítio na região de Águas Vermelhas/MG.

\section{MATERIAL E MÉTODOS}

O estudo foi conduzido em uma região do município de Águas Vermelhas, MG, localizado nas coordenadas geográficas $15^{\circ} 45^{\prime} 39^{\prime \prime}$ Sul e $41^{\circ} 33^{\prime} 42^{\prime \prime}$ Oeste. A altitude média é de $850 \mathrm{~m}$ com relevo variando entre plano a suave ondulado. Segundo a classificação de Koppen, o clima é tropical com estação seca de inverno (Aw), com temperatura e precipitação média anual variando entre 22 e $23^{\circ} \mathrm{C}$ e 702 a $882 \mathrm{~mm}$, respectivamente. Os solos da região são classificados como Latossolo Amarelo Distrófico (SOUZA et al., 2006)

Os dados foram obtidos em um plantio de Eucalyptus urophylla, com cinco anos de idade. Foram avaliados 3 clones de Eucalyptus urophylla S. T. Blake: AEC144, GG100 e AEC224, comumente destinados como matéria-prima para a produção de carvão vegetal. Os clones AEC144, GG100 e AEC224 estão registrados como cultivares no Ministério da Agricultura, Pecuária e Abastecimento, inscrição número 21874/2007, 21277/2006 e 21876/2007, respectivamente (MAPA, 2017).

A área da população em estudo correspondia a 36,67 ha divididos em 3 estratos. $O$ estrato 1 correspondente a 16,74 ha do clone AEC144, estrato 2 a 14,21 ha do clone GG 100 e o estrato 3 com 5, 71 ha do clone AEC224.

O espaçamento de plantio do povoamento foi de $3 \times 2,5 \mathrm{~m}^{2}$. Para a realização do inventário florestal utilizou-se o método de amostragem casual estratificada. $O$ formato das unidades amostrais utilizado foi quadrado com dimensão de $20 \times 20 \mathrm{~m}$ $\left(400 \mathrm{~m}^{2}\right)$. Foram lançadas cinco parcelas em cada um dos estratos dos clones AEC144 e GG100, e 3 parcelas na área do clone AEC224, totalizando uma intensidade amostral de $5200 \mathrm{~m}^{2}$, equivalente a $1,42 \%$. O erro de amostragem foi de $6,45 \%$, considerado aceitável pelos órgãos ambientais de Minas Gerais, demonstrando que o número de amostras foi suficiente em todos os extratos, visto que a intensidade amostral foi superior a $1 \%$ e o erro de amostragem foi menor que 10\% (SOARES et al., 2011).

Para a realização do inventário florestal foi medida a circunferência à altura do peito (CAP) e altura de todas as árvores da parcela. Para medir o CAP utilizou-se a fita métrica e para medir as alturas foi utilizado o hipsômetro de Suunto. Para a realização dos cálculos realizou-se a conversão do CAP para o diâmetro a altura do peito (DAP).

Realizou-se a distribuição diamétrica dos clones para orientar o procedimento de cubagem das árvores e posterior ajuste da equação volumétrica. $O$ método utilizado para a cubagem rigorosa foi o método de Smalian, sendo cubadas quatro árvores por classe de diâmetro por clone. A equação volumétrica utilizada foi a Equação 1 de Shumacher e Hall. 


$$
V=\beta_{0} \cdot D A P^{\beta_{1}} \cdot H^{\beta_{2}}
$$

em que:

$$
\begin{aligned}
& V=\text { volume } \\
& \text { DAP = diâmetro a altura do peito } \\
& H=\text { Altura }
\end{aligned}
$$

Realizou-se $o$ teste $t$ a $5 \%$ de erro de probabilidade para os seguintes parâmetros: média aritmética dos DAP's $(\mathrm{cm})$, diâmetro médio $(\mathrm{cm})$, área basal $\left(\mathrm{m}^{2} / \mathrm{ha}\right)$, volume $\left(\mathrm{m}^{3} / \mathrm{ha}\right)$, altura média $(\mathrm{m})$, incremento médio anual (IMA), número de indivíduos por hectare e volume médio por indivíduo.

\section{RESULTADOS E DISCUSSÃO}

$\mathrm{Na}$ tabela 1 estão as variáveis dendrométricas observadas nas parcelas amostrais para os três clones avaliados. Nota-se que o coeficiente de variação entre as parcelas foi maior para o volume médio por indivíduo $(14,88 \%)$ e volume por parcela $(14,65 \%)$, o que consequentemente influenciou a variância no incremento médio anual (IMA), oscilando entre 32,14 e $54,67 \mathrm{~m}^{3} / \mathrm{ha} /$ ano. Estes resultados demonstram uma heterogeneidade entre parcelas no que se refere ao volume de madeira por indivíduo, fato que pode ser explicado pela influência dos materiais genéticos ou devido a problemas de instalação no plantio do povoamento.

TABELA 1. Parâmetros dendrométricos mensurados nas parcelas amostrais.

\begin{tabular}{cccccccccc}
\hline Clone & Parcela & $\begin{array}{c}\text { DAP } \\
(\mathrm{cm})\end{array}$ & $\begin{array}{c}\mathrm{q} \\
(\mathrm{cm})\end{array}$ & $\mathrm{H}_{\text {média }}(\mathrm{m})$ & $\begin{array}{c}\text { Área } \\
\text { basal } \\
\left(\mathrm{m}^{2}\right)\end{array}$ & $\begin{array}{c}\text { Volume } \\
\left(\mathrm{m}^{3} / \text { parcela }\right)\end{array}$ & $\begin{array}{c}\text { No ind. / } \\
\text { parcela }\end{array}$ & $\begin{array}{c}\text { IMA } \\
\left(\mathrm{m}^{3} / \mathrm{ha} / \mathrm{ano}\right)\end{array}$ & $\begin{array}{c}\mathrm{V}_{\text {médio }} \\
\text { /ind. } \\
\left(\mathrm{m}^{3}\right)\end{array}$ \\
\hline AEC144 & 1 & 13,27 & 13,36 & 19,00 & 0,7431 & 8,35 & 52 & 41,78 & 0,1607 \\
AEC144 & 2 & 14,29 & 14,21 & 19,26 & 0,8115 & 9,10 & 52 & 45,52 & 0,1751 \\
AEC144 & 3 & 14,90 & 14,96 & 19,65 & 0,9839 & 10,93 & 55 & 54,67 & 0,1988 \\
AEC144 & 4 & 12,84 & 13,17 & 17,04 & 0,8037 & 8,35 & 59 & 41,77 & 0,1416 \\
AEC144 & 5 & 13,50 & 13,45 & 18,07 & 0,8520 & 9,06 & 60 & 45,32 & 0,1510 \\
GG100 & 6 & 12,56 & 12,63 & 15,49 & 0,6769 & 6,42 & 54 & 32,14 & 0,1190 \\
GG100 & 7 & 12,58 & 12,69 & 17,05 & 0,6958 & 7,15 & 55 & 35,77 & 0,1300 \\
GG100 & 8 & 12,98 & 13,10 & 17,05 & 0,6958 & 7,58 & 56 & 37,91 & 0,1353 \\
GG100 & 9 & 12,91 & 12,95 & 17,66 & 0,7635 & 7,97 & 58 & 39,89 & 0,1375 \\
GG100 & 10 & 12,82 & 12,89 & 17,96 & 0,7306 & 7,76 & 55 & 38,83 & 0,1412 \\
AEC224 & 11 & 13,33 & 13,51 & 18,48 & 0,7456 & 7,83 & 52 & 39,16 & 0,1506 \\
AEC224 & 12 & 12,60 & 12,71 & 18,09 & 0,6220 & 6,51 & 49 & 32,59 & 0,1330 \\
AEC224 & 13 & 14,01 & 14,42 & 18,99 & 0,8166 & 8,63 & 49 & 43,19 & 0,1763 \\
\hline Média & - & 13,28 & 13,39 & 17,98 & 0,76 & 8,13 & 54,31 & 40,66 & 0,15 \\
Desvio & - & 0,72 & 0,72 & 1,14 & 0,09 & 1,19 & 3,47 & 5,95 & 0,02 \\
CV(\%) & - & 5,46 & 5,42 & 6,35 & 12,03 & 14,65 & 6,40 & 14,65 & 14,88 \\
\hline Em que
\end{tabular}

Em que: $\mathrm{DAP}_{\text {médio }}=$ média aritmética dos diâmetros; $q=$ diâmetro médio; $\mathrm{H}_{\text {média }}=$ altura média; $\mathrm{V}_{\text {médio }}=$ volume médio por parcela; $\mathrm{V}_{\text {médio }} /$ ind. = volume médio por indivíduo; IMA = incremento médio anual; $\mathrm{CV}=$ coeficiente de variação

$\mathrm{Na}$ comparação entre as médias dos clones realizada a partir do teste $\mathrm{t}$ foi observada diferença significativa para todas as variáveis estimadas $(p<5 \%)$ (Tabela 2). Os resultados encontrados refletem uma boa adaptação dos clones a região de plantio. O incremento médio anual de $40,34 \mathrm{~m}^{3} / \mathrm{ha}$ /ano do povoamento para a região de Águas Vermelhas no Norte de Minas Gerais pode ser considerado um crescimento bom, uma vez que se aproxima dos níveis considerados altos (45-60 
$\mathrm{m}^{3} / \mathrm{ha}$ /ano) por Higashi et al. (2000). Os dados deste trabalho também foi superior ao estimado para a região por Guimarães et al. (2007) que foi entre 30 e 35 m³/ha/ano.

TABELA 2. Valores das variáveis dendrométricas estimadas para os três clones avaliados.

\begin{tabular}{ccccccccc}
\hline & $\begin{array}{c}\text { DAP } \\
(\mathrm{cm})\end{array}$ & $\mathrm{q}(\mathrm{cm})$ & $\begin{array}{c}\mathrm{H}_{\text {média }} \\
(\mathrm{m})\end{array}$ & $\begin{array}{c}\text { Área basal } \\
\left(\mathrm{m}^{2} / \mathrm{ha}\right)\end{array}$ & $\begin{array}{c}\text { Volume } \\
\left(\mathrm{m}^{3} / \mathrm{ha}\right)\end{array}$ & $\begin{array}{c}\text { № ind./ } \\
\text { há }\end{array}$ & $\begin{array}{c}\text { IMA } \\
\left(\mathrm{m}^{3} / \mathrm{ha} / \mathrm{an}\right. \\
\mathrm{o})\end{array}$ & $\begin{array}{c}\mathrm{V}_{\text {médio }} \\
\text { /ind. }\left(\mathrm{m}^{3}\right)\end{array}$ \\
\hline AEC144 & $13,62 \mathrm{a}$ & $13,78 \mathrm{a}$ & $18,47 \mathrm{ab}$ & $20,97 \mathrm{a}$ & $229,09 \mathrm{a}$ & $1390 \mathrm{a}$ & $45,81 \mathrm{a}$ & $0,1648 \mathrm{a}$ \\
GG 100 & $12,77 \mathrm{~b}$ & $12,85 \mathrm{~b}$ & $17,04 \mathrm{~b}$ & $17,81 \mathrm{~b}$ & $184,56 \mathrm{~b}$ & $1390 \mathrm{a}$ & $36,91 \mathrm{~b}$ & $0,1327 \mathrm{~b}$ \\
AEC 224 & $13,31 \mathrm{ab}$ & $13,55 \mathrm{ab}$ & $18,52 \mathrm{a}$ & $18,20 \mathrm{ab}$ & $191,58 \mathrm{ab}$ & $1250 \mathrm{~b}$ & $38,31 \mathrm{ab}$ & $0,1532 \mathrm{~b}$ \\
\hline Média & 13,28 & 13,41 & 18,06 & 19,00 & 201,75 & 1343,33 & 40,34 & 0,15 \\
Desvio & 0,49 & 0,50 & 0,88 & 1,72 & 23,94 & 80,83 & 4,79 & 0,02 \\
CV & 3,72 & 3,75 & 4,86 & 9,07 & 11,87 & 6,02 & 11,87 & 10,80 \\
\hline
\end{tabular}

Em que: $\mathrm{DAP}_{\text {médio }}=$ média aritmética dos diâmetros; $\mathrm{q}=$ diâmetro médio; $\mathrm{H}_{\text {média }}=$ altura média; $\mathrm{V}_{\text {médio }}=$ volume médio por parcela; $\mathrm{V}_{\text {médio }}$ / ind. = volume médio por indivíduo; IMA = incremento médio anual; $\mathrm{CV}=$ coeficiente de variação

A média aritmética dos diâmetros e o diâmetro médio foram maior no clone AEC 144, que foi significativamente superior ao clone GG 100 e ambos não diferiram do clone AEC 224. Como a estimativa volumétrica é função dos dados de diâmetro e altura estes influenciaram no cálculo do volume, apresentando os mesmos resultados.

A altura do clone AEC 144 mostrou-se superior em relação ao clone GG100, mas estatisticamente semelhante ao clone AEC 224, onde as médias diferiram apenas em 5 centímetros. Protásio et al. (2014), avaliando as características da madeira de clones comerciais de Eucalyptus spp. visando o uso bioenergético relataram que o clone AEC 144 destacou quanto as características de diâmetro à altura do peito (DAP) e altura total. Para estes autores essas duas características são fundamentais para a determinação do volume de madeira e consequentemente, influencia na produtividade dos clones de eucalipto.

Ao analisar os dados de área basal verificou-se os mesmos resultados do diâmetro médio. O clone AEC144 foi significativamente superior ao clone GG 100 e ambos não diferiram do clone AEC 224. A variável área basal é importante para definir a intensidade do desbaste, e pode ser considerado o melhor critério para a tomada de decisão de um possível desbate no povoamento (CAMPOS; LEITE, 2013). A opção de realizar um desbaste no clone AEC144 seria viável e proporcionaria maior quantidade de madeira em relação aos demais clones, principalmente por seu diâmetro médio ser superior.

Para o número de indivíduos por hectare os resultados mostraram que os clones AEC144 e GG100 obtiveram o mesmo desempenho, já o clone AEC 224 foi estatisticamente inferior. Uma das possíveis causas para o clone AEC 224 ter apresentado um número de 140 indivíduos a menos que os demais podem estar relacionado com a mortalidade após o plantio ou falhas no espaçamento entre árvores. As causas de mortalidade das mudas na fase inicial de crescimento geralmente estão relacionadas com o estresse hídrico, mas também são comuns problemas com formigas cortadeiras, cupins e gradiente de fertilidade, sendo primordial boas práticas de manejo, principalmente na fase estabelecimento da cultura (REIS et al., 2015; SILVA et al., 2015).

Os dados de volume/ha e incremento médio anual (IMA) apresentaram resultados semelhantes à área basal e diâmetro médio. O clone AEC144 e o AEC ENCICLOPÉDIA BIOSFERA, Centro Científico Conhecer - Goiânia, v.14 n.26; p.79 2017 
224 foi superior ao clone GG100, demonstrando uma maior eficiência no aproveitamento dos fatores que influenciam 0 crescimento e adaptação às condições edafoclimáticas locais. Verificou-se que apesar dos clones AEC144 e o AEC224 serem estatisticamente semelhante, o clone AEC144 apresentou maiores valores, obtendo 229,09 $\mathrm{m}^{3} /$ ha de volume enquanto o AEC 224 191,58 m³/ha.

Para selecionar espécies em plantios comerciais a variável mais utilizada é o volume, sendo o incremento médio anual (IMA) o primeiro indicador do potencial da espécie (TONINI et al., 2006). O IMA de 45,81 $\mathrm{m}^{3} / \mathrm{ha} / \mathrm{ano}$ do clone AEC 144 demonstra um bom crescimento indicando uma boa adaptação do clone para a região em estudo.

Reis et al. (2014), avaliando o desempenho dos diferentes clones de eucaliptos em uma região de Ponta Porã, Mato Grosso do Sul relataram que os clones de maior desempenho, em volume de madeira foram AEC 144 e AEC 224, além do bom crescimento, também apresentaram as melhores notas em termos de forma do fuste, espessura de galhos, desrama natural, baixa presença ou ausência de bifurcações, doenças e insetos-praga. Ambos pertencem à espécie Eucalyptus urophylla e se caracterizam pelo bom desempenho e alta adaptabilidade em diversas regiões mesmo quando cultivado em locais de deficiência hídrica e elevadas temperaturas (FONSECA et al., 2010)

Em estudos realizados por Cipriani et al. (2013) sobre o crescimento inicial de clones de eucalipto no município de Vilhena - Rondônia relataram o crescimento do clone I144 e do GG100, por apresentarem IMA superior a $40 \mathrm{~m}^{3} / \mathrm{ha} /$ ano aos 37 meses demonstrando um crescimento satisfatório desses materiais genéticos.

As estimativas de número de indivíduos, por clone e por classe de diâmetro encontra-se na Figura 1. De forma geral, o maior número de árvores concentrou-se nas classes diamétricas de 12 e $14 \mathrm{~cm}$ em todos os clones, demonstrando que 0 povoamento apresentou uma distribuição normal, ou seja, menor número de indivíduos nas classes extremas (6-8 e 18-20) e uma maior concentração dos indivíduos nas classes mais próximas à média.

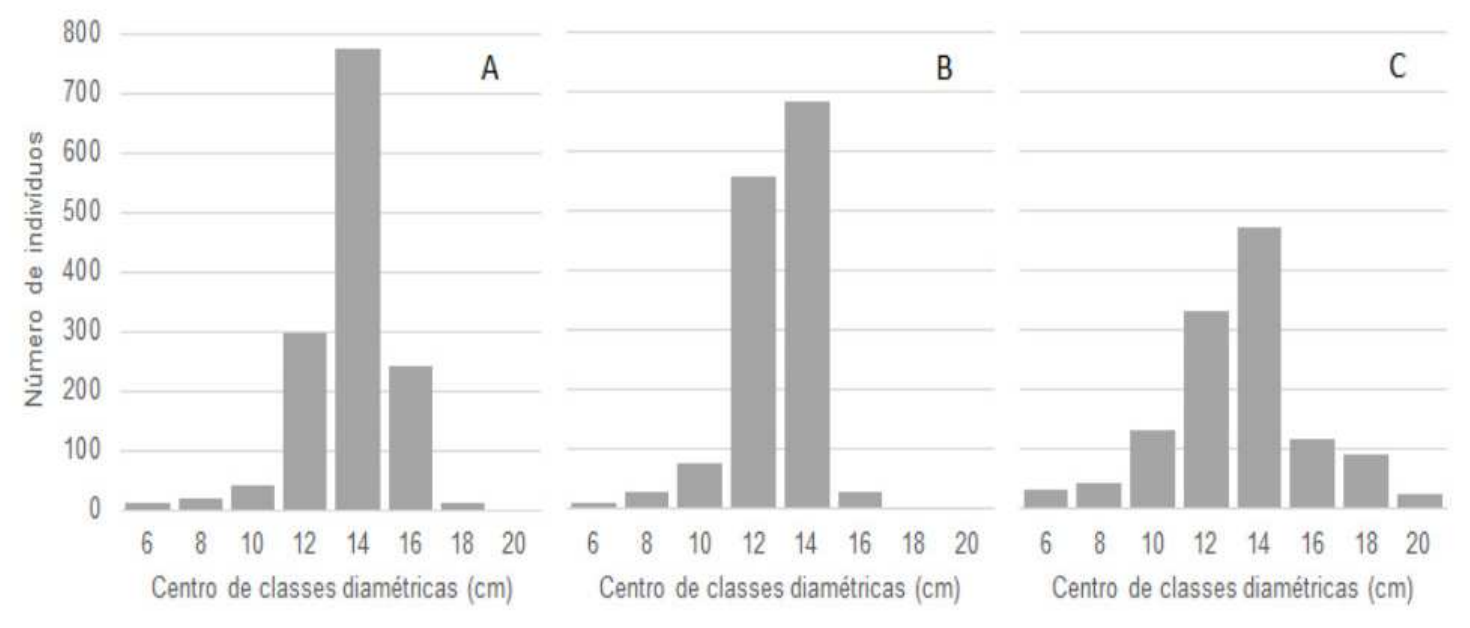

FIGURA 1 - Distribuição do número de indivíduos do clone AEC 144 por classe de diâmetro $(A)$; distribuição do número de indivíduos do clone GG100 por classe de diâmetro (B); distribuição do número de indivíduos do clone AEC 224 por classe de diâmetro (C). 
A uniformidade dos plantios dos clones avaliados (Figura 1), é uma característica marcante da silvicultura clonal em plantações de eucalipto, o que possibilita maior controle sobre a qualidade dos produtos; maximização em ganho em produtividade silvicultural; aumenta a eficiência nos tratos sulviculturais de poda e desbastes; além de facilitar as operações mecanizáveis de adubação e controle plantas espontâneas, pragas e doenças (XAVIER et al., 2013).

As estimativas do volume $\left(\mathrm{m}^{3} / \mathrm{ha}\right)$ por clone e por classe de diâmetro (Tabela 3) seguem a mesma tendência da distribuição diamétrica por número de indivíduos. O clone AEC144 teve $57,24 \%$ de seu volume concentrado na classe de diâmetro central (classe 14) e o clone GG100 teve 56,59\%, enquanto o clone AEC 224 teve apenas $41,15 \%$ de seu volume nesta classe. Estes dados demonstram uma disparidade entre estes clones na concentração volumétrica por classe de diâmetro. Fato explicado pela diferença no número de indivíduos do clone AEC 224.

TABELA 3. Estimativas de volume do fuste $\left(m^{3} / \mathrm{ha}\right)$, por clone, por classe de diâmetro

\begin{tabular}{cccccccccc}
\hline & \multicolumn{10}{c}{ Centro de classe de diâmetro (cm) } \\
\hline Clone & 6 & 8 & 10 & 12 & 14 & 16 & 18 & 20 & Total \\
\hline AEC144 & 0,42 & 1,27 & 4,26 & 38,16 & 131,14 & 50,99 & 2,98 & 0 & 229,09 \\
GG100 & 0,29 & 1,41 & 5,82 & 67,08 & 104,45 & 5,48 & 0 & 0 & 184,56 \\
AEC224 & 0,95 & 2,18 & 11,33 & 41,05 & 78,85 & 24,21 & 24,23 & 8,75 & 191,58 \\
\hline Total & 1,66 & 4,88 & 21,42 & 146,30 & 314,45 & 80,69 & 27,22 & 8,75 & 605,23 \\
\hline
\end{tabular}

Por outro lado é possível observar que apesar do clone AEC 224 ter um menor número de indivíduos por classe diamétrica (Figura 1), este apresentou melhor distribuição de indivíduos entre as classes de diâmetro. Quando se pensa em destino para a produção de madeira deste povoamento pode-se dizer que o clone AEC 224 demonstrou um bom potencial para o uso múltiplo, visto que possui mais indivíduos nas menores e maiores classes que os demais clones (Figura 1C). Os clones AEC 144 e GG 100 tiveram maior concentração de indivíduos nas classes centrais, mostrando a homogeneidade de crescimento e seu potencial para um produto com diâmetro padrão (Figura 1A e 1B).

\section{CONCLUSÃO}

Os clones de Eucalyptus urophylla tiveram incremento médio anual (IMA) satisfatório quando comparado a resultados obtidos na mesma região de estudo, demonstrando boa adaptação às condições ambientais. Desta forma, o uso desses clones em plantios comerciais pode proporcionar boa produtividade, desde que aplicadas adequadas técnicas silviculturais.

O clone AEC144 e o AEC224 apresentaram resultados semelhantes para a maioria das variáveis estimadas, sendo os mais recomendados para o plantio na região norte de Minas Gerais.

\section{REFERÊNCIAS}

CAMPOS, J. C. C.; LEITE, H. G. Mensuração florestal: perguntas e respostas. $4^{\text {a }}$ Ed. Viçosa: Ed. UFV, 2013, 605p. 
CIPRIANI, H. N; VIEIRA, A. H.; GODINHO, V. P. C. Crescimento inicial de clones de eucalipto em Vilhena, RO. Embrapa Rondônia-Comunicado Técnico (INFOTECAE), 2013.

FERNANDES, A. Biodiversidade da caatinga. In: ARAUJO, E. L.; MOURA, A. N.; SAMPAIO, E. V. S. B.; GESTINARI, L. M. S.; CARNEIRO, J. M. T. (Ed.). Biodiversidade, conservação e uso sustentável da flora do Brasil. Recife: UFRPE e SBB, 2002. p. 42-43.

FONSECA, S. M.; RESENDE, M. D. V.; ALFENAS, A. C.; GUIMARÃES, L. M. S.; ASSIS, T. F.; GRATTAPAGLIA, D. Manual Prático de Melhoramento Genético do Eucalipto. Viçosa, UFV, 2010, 200p.

GUIMARÃES, D. P.; SILVA, G. G. C.; SANS, L. M. A.; LEITE, F. P. Uso do modelo 3-PG para o zoneamento do potencial produtivo do eucalipto no estado de Minas Gerais. Revista Brasileira de Agrometeorologia, v.15, n.2, p. 192-197, 2007. Disponível em: http://ainfo.cnptia.embrapa.br/digital/bitstream/item/66837/1/Usomodelo.pdf.

HIGASHI, E. N.; SILVEIRA, R. L. V. A.; GONÇALVES, A. N. Propagação vegetativa de Eucalyptus: princípios básicos e a sua evolução no Brasil. Circular Técnica IPEF, n. 192, p.11, 2000.

IBA - Indústria Brasileira de Árvores. Anuário Estatístico 2017. Brasília, DF, 2017. $80 \mathrm{p}$.

MAPA - Ministério da Agricultura, Pecuária e Abastecimento. Registro Nacional de Cultivares - RNC. Disponível em: http://www.agricultura.gov.br/guia-deservicos/registro-nacional-de-cultivares-rnc. acesso em: 10 de setembro de 2017.

PROTÁSIO, T. P.; GOULART, S. L.; NEVES, T. A.; ASSIS, M. R.; TRUGILHO, P. F. Clones comerciais de Eucalyptus de diferentes idades para o uso bioenergético da madeira. Scientia florestalis, Piracicaba, v. 42, n.101, p. 113-127, 2014. Disponível em: http://dx.doi.org/10.5902/1980509814587.

REIS, C. A. F.; SANTOS, P. E. T.; PALUDZYSZYN FILHO, E. Avaliação de clones e eucalipto em Ponta Porã, Mato Grosso do Sul. Pesquisa Florestal Brasileira, Colombo, v. 34, n. 80, p. 263-269, 2014. Disponível em: http://dx.doi.org/10.4336/2014.pfb.34.80.569.

REIS, M. A.; CUNHA, J. P. A. R.; ZANETTI, R.; FERNANDES, B. V.; REIS, J. M. R. aplicação sistemática mecanizada de isca formicida granulada em eucaliptais em fase de manutenção. Cerne, v. 21 n. 3 | p. 423-428, 2015. Disponível em: <http://www.redalyc.org/articulo.oa?id=74442824010> doi: 10.1590/0104776020152103133

ROSAdO, M. A.; ROSADO, T. B.; ALVES, A. A.; LAVIOLA, A. M.; BHERING, L. L. Seleção simultânea de clones de eucalipto de acordo com produtividade, estabilidade e adaptabilidade. Pesquisa Agropecuária Brasileira, Brasília, v.47, 
n.7, p.964-971, 2012. Disponível em: http://dx.doi.org/10.1590/S0100204X2012000700013.

SILVA, A. N. T.; CUNHA, H. F.; RICARDO, J. A. D.; ABOT, A. R. Espécies de cupins (isoptera) em cultura de eucalipto sob diferentes sistemas de manejo de irrigação, em região de transição cerrado-pantanal de Mato Grosso do Sul, Brasil.. Revista Árvore, Viçosa-MG, v. 39, n. 1, p.137-146, 2015. Disponível em: http://dx.doi.org/10.1590/0100-67622015000100013.

SOARES, C. P. B.; PAULA NETO, F. P.; SOUZA, A. L. Dendrometria e Inventário Florestal. 2ª . ed. Viçosa, MG: Editora UFV, 2011. 272p.

SOUZA, M. J. H.; GUIMARÃES, M. C. A.; GUIMARÃES, C. D. L.; FREITAS, W. S.; OLIVEIRA, A. M. S. Potencial agroclimático para a cultura da acerola no Estado de Minas Gerais. Revista Brasileira de Engenharia Agrícola e Ambiental, v.10, n.2, p.390-396, 2006. Disponível em: http://dx.doi.org/10.1590/S1415 43662006000200021.

TAIZ, L.; ZEIGER, E. Fisiologia vegetal. 5. ed. Porto Alegre: Artmed, 2013. 954p.

TATAGIBA, S. D.; PEZZOPANE, J. E. M.; VINCO, J. S.; PINHEIRO, A. A. Crescimento de clones de eucalipto em diferentes condições microclimáticas e lâminas de água no substrato. Irriga, Botucatu, v. 21, n. 1, p. 104-118, janeiromarço, 2016. Disponível em: http://dx.doi.org/10.15809/irriga.2016v21n1p104-118.

TATAGIBA, S. D.; XAVIER, T. M. T.; TORRES, H.; PEZZOPANE, J. E. M.; CECÍLIO, R. A.; ZANETTI, S. S. Determinação da máxima capacidade de retenção de água no substrato para produção de mudas de eucalipto em viveiro. Floresta, Curitiba, v.45, n. 4, p. 745-754, 2015. Disponível em: http://dx.doi.org/10.5380/rf.v45i4.38334.

TONINI, H.; ARCO-VERDE, M. F.; SCHWENGBER, D.; MOURÃO JUNIOR, M. Avaliação de espécies florestais em área de mata no estado de Roraima. Cerne, v. 12, n. 1, 2006. Disponível em: http://www.redalyc.org/articulo.oa?id=74412102> ISSN 0104-7760.

VENTURIN, N.; CAMPINHOS JR., E.; MACEDO, R. L. G.; VENTURIN, R. P. Histórico. In: VALE, A. B. ; MACHADO, C. C.; PIRES, J. M. M.; VILAR, M. V.; COSTA, C. B.; NACIF, A. P. (Org.). Eucalipto cultura no Brasil: silvicultura, manejo e ambiência. Viçosa, MG: SIF, 2014. p. 17-38.

XAVIER, T. M. T.; PEZZOPANE, J. E. M.; PENCHEL R. M.; CALDEIRA M. V. W.; REIS, F. R. Influence of water deficit and season on biomass yield from clonal stands of Eucalyptus. Cerne, Lavras, v. 19, n. 3, p. 473-479, 2013. Disponível em: http://dx.doi.org/10.1590/S0104-77602013000300015.

XAVIER, A.; WENDLING, I.; SILVA, R. L. da. Silvicultura Clonal: princípios e técnicas. Viçosa, MG: Editora UFV, 2013. 279 p. 